\title{
Three-Flavor Symmetry of Hadrons Consistent With The Okubo-Zweig-Iizuka Rule
}

\author{
M. Kirchbach * \\ Escuela de Fisica, Univ. Aut. de Zacatecas, \\ Apartado Postal C-580, Zacatecas \\ ZAC 98068, Mexico
}

August 27, 2018

\begin{abstract}
It is argued that the only three-flavor symmetry which is consistent with the Okubo-Zweig-Iizuka (OZI) rule, and is therefore dictated by the gluon gauge dynamics, is the heavy $c$ quark limit of a certain $\mathrm{U}(4)_{F}$ subgroup (called $\mathrm{S}_{O Z I}$ here) and defined as $\mathrm{S}_{O Z I}=\lim _{m_{c} \rightarrow \Lambda_{c}}$ $\mathrm{SU}(2)_{u d} \otimes \mathrm{SU}(2)_{c d} \otimes \mathrm{U}(1)$ with the two $\mathrm{SU}(2)$ groups acting in turn onto the 1st and 2nd quark generations rather than Gell-Mann's eightfold $\mathrm{SU}(3)_{F}$. Within this scheme the presence of non-strange quarkonium components in the wave function of the physical $\eta$ and $f_{1}(1285)$ mesons appears necessarily through the violation of the OZI rule for the pseudoscalar and axial vector mesons by the anomalous axial $\mathrm{U}(1)_{A}$ baryon number current and not by a primordial octet flavor symmetry. The physical observable which selects the $\mathrm{S}_{O Z I}$ over the $\mathrm{SU}(3)_{F}$ scheme is the non-isotriplet part of the neutral axial current. In the case of the $S_{O Z I}$ symmetry the only non-isotriplet neutral axial current having a well defined chiral limit is purely strange, while within the $\mathrm{SU}(3)_{F}$ framework it is the octet axial current which is supposed to be partially conserved. Accordingly, while in the latter case the tree level $\eta N$ coupling is of significant strength, its almost vanishes in the former, in agreement with data, thus confirming the relevance of the $\mathrm{S}_{O Z I}$ symmetry.
\end{abstract}

\footnotetext{
${ }^{*}$ On leave of absence from Institut für Kernphysik, Universität Mainz, D-55099 Mainz, Germany.
} 


\section{Introduction}

The idea that the three-flavor symmetry of hadrons is governed by the group $\mathrm{SU}(3)_{F}$ is one of the ruling concepts of strong interaction physics. It has its roots in the empirical observation that the lightest mesons constitute a family of eight approximately mass degenerate particles of equal spins and parities but of different electric and strangeness charges. Same is valid for the lightest spin- $1 / 2$ baryons, while the spin- $3 / 2$ baryons join to a decuplet. To explain such patterns, the existence of the three fundamental subbaryon degrees of freedom with fractional electric charges, and approximately equal masses, the $u_{-}, d_{-}$, and $s$ quarks (in the established notation), has been concluded by Zweig [1] and Gell-Mann [2] in the early sixties. Indeed, in disregrading the order of the three quarks constituting a baryon and in using simplest combinatorics laws, one finds ten essentially different three quark (3q) configurations to exist. These consist of, a) the three sameflavor combinations uuu, $d d d$, and sss, b) the six combinations uud, uus, $d d u, d d s, s s u$, and $s s d$ in which the same flavor appears only twice, and c) the single configuration $u d s$ where all three quarks carry different flavors. The ten configurations are in turn identified with the spin-3/2 particles $\Delta^{++}, \Delta^{-}, \Omega^{-}, \Delta^{+}, \Sigma^{*+}, \Delta^{0}, \Sigma^{*-}, \Xi^{* 0}, \Xi^{*-}$, and $\Sigma^{* 0}$, respectively. From this decuplet family one easily deduces the existence of lower dimensional pattern by first amputating the $\Delta^{++}, \Delta^{-}$, and $\Omega^{-}$decuplet 'edges', then replacing $\Delta^{+}$by the proton $(p), \Delta^{0}$ by the neutron $(n)$, and by removing the asterisk from the remaining hyperons to account for the lower spin-1/2 of the new $3 q$ configurations. To obtain the octet one finally has to account for the double occupation of the $(u d s)$ configuration by the physical $\Sigma^{0}$ and $\Lambda$ hyperons corresponding to a neutral charge triplet- and a genuine charge singlet states, respectively.

The appearance of the octet and the decuplet patterns in the spectroscopy of hadrons with low masses is therefore primarily the direct consequence of the relevance of the three fundamental subbaryon degrees of freedom. As a next step, one has to select a mathematical structure suited for the description of these patterns. In the early sixties, when the knowledge about the existence of further flavors, the quark generations, and the anomaly of the axial currents was still absent, it appeared natural to assume that the three quark degrees of freedom may transform according to the fundamental representation of the unitary group $\mathrm{U}(3)$ and that the octet and the decuplet may be associated with representations of its special form $\mathrm{SU}(3)$. Through this step, an ansatz for the flavor symmetry of low mass 
spectroscopy was made and simultaneously identified with the symmetry of the strong interaction lagrangian.

This identification is not as harmless as it looks, in particular because the mere existence of a regularity in the spectroscopic patterns of the lightest hadrons is not sufficient to trace back in an unique way its symmetry origin. A possible flaw of the symmetry ansatz for the low energy sector can easily be transferred to both the excitation spectra and the dynamics of hadrons and create problems at a later stage. Indeed, the symmetry of the lagrangian determines besides the properties of the states near vacuum also the hadron excitation spectra. Moreover, under certain circumstances, this symmetry can put strong constraints on the hadron dynamics. For example, in the case of a symmetry realized in the Nambu-Goldstone mode, the interaction is determined by the exchange of the corresponding Goldstone bosons between the matter fields with the boson-matter field couplings being prescribed by the symmetry considered. In assuming, therefore, the symmetry of the strong interaction lagrangian to be $\mathrm{SU}(3)_{F}$, one first expects the appearance of octet and decuplet pattern for excited baryons. This is not unproblematic. It has been shown in a series of papers [3] that the baryon excitation spectra reveal a systematic Lorentz covariant spin clustering with the spin clusters transforming in accordance to finite dimensional $\mathrm{SU}(2)_{I} \otimes \mathrm{O}(1,3)$ multiplets. This clustering of baryon resonances, in which the nucleon and $\Delta$ spectra below $2000 \mathrm{MeV}$ are complete, is incompatible with the $\left[\mathrm{SU}(3)_{F} \otimes \mathrm{SU}(2)_{S}\right] \times \mathrm{O}(3)_{L}$ classification scheme with its numerous unobserved 'missing' resonances. A second inevitable consequence of the $\mathrm{SU}(3)_{F}$ symmetry ansatz concerns the chiral symmetry $\mathrm{SU}(3)_{L} \otimes \mathrm{SU}(3)_{R}$ of $\mathrm{QCD}$, the gauge theory of strong interaction. Chiral symmetry is ordinarily supposed to be the global internal symmetry of the QCD lagrangian and is considered to be realized in the non-multiplet Nambu-Goldstone mode with the eight lightest pseudoscalar mesons being associated with the expected Goldstone bosons. Within this scheme with conserved vector currents, the axial ones can mostly be partially conserved if the tree level meson-baryon coupling constants are predetermined by means of the corresponding Goldberger-Treiman (GT) relations 《4. As long as such couplings can be extracted from either meson-nucleon scattering cross sections, or from measuring the meson production rates, off, say, a proton target, a direct comparison to data can be performed. As a result of such a comparison, one finds that the pion-nucleon (isovector) GT relation is valid to quite a good accuracy [5], while the octet one, predicting the $\eta N$ coupling, is seriously violated [6]. Data are compatible with a decoupling of 
the $\eta$ meson from nucleons and hyperons, rather than with its supposed octet Goldstone boson nature (see Ref. [6]) for a recent review). The experience made with the eightfold way over the last three decades shows that there are several aspects of hadron physics which appear incompatible with the $\mathrm{SU}(3)_{F}$ scheme. Because of that, gradually and gradually the impression arises that it is timely to revisit the $\mathrm{SU}(3)_{F}$ concept by incorporating the contemporary knowledge about the existence of the three quark generations, the relevance of color gauge dynamics and the axial current anomaly problems for hadron systems. In other words, it appears timely to require three-flavor symmetry to arise from a symmetry acting within a flavor space containing all three, or at least two, complete quark generations after freezing out the heavy flavor degrees of freedom. We here show that the three-flavor symmetry obtained from the four flavor one after freezing out the $c$ quark, deviates from the eightfold $\mathrm{SU}(3)_{F}$ especially with respect to the structure of the non-isovector part of the neutral axial current and resolves the problem of the observed strong suppression of the $\eta N N$ vertex over the $\pi N N$ one. We find the precise three-flavor symmetry of hadrons to be the heavy $c$ quark limit of a certain subgroup, called $\mathrm{S}_{O Z I}$ here, of the four-flavor unitary group $\mathrm{U}(4)_{F}$, and defined as

$$
S_{O Z I}=\lim _{m_{c} \rightarrow \Lambda_{c}} S U(2)_{u d} \otimes S U(2)_{c s} \otimes U(1),
$$

where the two $\mathrm{SU}(2)$ groups act in turn onto the first and second quark generations.

The new group symmetry has several advantages over $\mathrm{SU}(3)_{F}$. First of all it shares common features with the anomaly free SU(4) theory as it acts onto two complete quark generations. Second, it respects the gluon gauge dynamics in so far that it respects the Okubo-Zweig-Iizuka (OZI) rule [7] by predicting the correct spectroscopic properties of the mesons in the anomaly free vector sector. We finally conclude that the precise three flavor chiral symmetry of hadrons is $\mathrm{S}_{O Z I}^{L} \otimes \mathrm{S}_{O Z I}^{R}$ and emphasize that the $\mathrm{S}_{O Z I}$ Goldstone bosons are the pions and the kaons, while the $\eta$ meson behaves as a masked strange Goldstone boson, a result already conjectured in the previous work [6]. 


\section{The $\mathrm{S}_{O Z I}$ group- the new three-flavor symmetry of hadrons compatible with the OZI rule}

The main flaw of the $\mathrm{SU}(3)_{F}$ ansatz is related to the choice for the representation of the second diagonal $\mathrm{SU}(3)_{F}$ generator, known as the octet one, and denoted by

$$
\lambda^{8}=1 / \sqrt{3} \operatorname{diag}(1,1,-2) .
$$

Here, $\lambda^{8}$ has been supposed to act onto the three flavor space $q_{3}=$ column $(\mathrm{u}, \mathrm{d}, \mathrm{s})$ [2, 8]. The major physical observable associated with $\lambda^{8}$ is the wave function of the so called octet eta meson, here denoted by $\left|\eta_{8}\right\rangle$, and given below as

$$
\left|\eta_{8}\right\rangle=\sqrt{2} \bar{q}_{3} \frac{\lambda^{8}}{2} q_{3}
$$

The $\eta_{8}$ meson is one of the three mesons occupying the center of the pseudoscalar meson octet and its wave function is obtained in the course of the orthonormalization procedure of these neutral $\mathrm{U}(3)_{F}$ states. The first such state is the neutral pion, $\left|\pi^{0}\right\rangle$, the member of the charge triplet, and its structure is determined in an unique way from the properties of the corresponding ladder operators $T_{ \pm}$

$$
\sqrt{2}\left|\pi^{0}\right\rangle=T_{ \pm}\left|\pi^{\mp}\right\rangle, \quad \pi^{+}=\bar{d} u, \quad \pi^{-}=-d \bar{u}
$$

as

$$
\begin{aligned}
\left|\pi^{0}\right\rangle=\sqrt{2} \bar{q}_{3} \frac{\lambda^{3}}{2} q_{3} & =\frac{1}{\sqrt{2}}(\bar{u} u-\bar{d} d) \\
\lambda^{3} & =\operatorname{diag}(1,-1,0) .
\end{aligned}
$$

In presupposing the second neutral state to be the $\mathrm{U}(1)_{F}$ singlet

$$
\begin{aligned}
\left|\eta_{1}^{F}\right\rangle=\frac{1}{\sqrt{3}}(\bar{u} u+\bar{d} d+\bar{s} s), & =\sqrt{2} \bar{q}_{3} \frac{\lambda^{0}}{2} q_{3}, \\
\lambda^{0} & =\sqrt{\frac{2}{3}} \mathbb{1}_{3},
\end{aligned}
$$

the octet state in Eq. (3) is constructed as the only state orthogonal to the previous two. In this way Gell-Mann's eightfold way choice for the diagonal $\mathrm{U}(3)_{F}$ generators $\lambda^{3}, \lambda^{8}$, and $\lambda^{0}$ has been made. 
This choice is by no means unique and needs be confirmed by the data. In general, $\mathrm{SU}(\mathrm{N})$ state can equally well be described in terms of the new set of diagonal flavor generators

$$
\left(E_{k k}\right)_{i j}=\delta_{k i} \delta_{k j}-\delta_{k+1, i} \delta_{k+1, j}, \quad k=1, \ldots, N-1, \quad i, j=1, \ldots, N .
$$

For concreteness, for $\mathrm{SU}(3)$ one finds $\mathrm{E}_{11}=\operatorname{diag}(1,-1,0)$, and $\mathrm{E}_{22}=\operatorname{diag}(0,1,-$ $1)$. This is the so called Weyl's choice for the $\mathrm{su}(3)_{F}$ algebra [9]. Note, that the matrix $\lambda^{3}$ coincides with $\mathrm{E}_{11}$ while $\lambda^{8}=\left(\mathrm{E}_{11}+2 \mathrm{E}_{22}\right) / \sqrt{3}$. Another set of diagonal orthogonal $\mathrm{U}(3)_{F}$ states can be constructed by using instead of the three-flavor singlet from above, the isospin (I) singlet

$$
\left|\eta_{1}^{I}\right\rangle=\frac{1}{\sqrt{2}}(\bar{u} u+\bar{d} d) .
$$

Then the remaining thrid state that is orthogonal to both the $\left|\pi^{0}\right\rangle$ and $\left|\eta_{1}^{I}\right\rangle$ states is the purely strange quarkonium

$$
\left|\eta^{s}\right\rangle=-\bar{s} s
$$

These examples show that neither the choice for the neutral states, nor the choice for the flavor generators is unique but it needs further specification by comparison with data. Mainly, one compares the masses of the mathematical with the physical hadron states in terms of the well known mass formulae (compare [8] for details). In doing so, one finds the wave functions of the physical $\eta$ meson (here denoted by $|\eta\rangle$ ) to slightly deviate from the octet state according to 10

$$
\begin{aligned}
|\eta\rangle & =\alpha\left|\eta_{8}\right\rangle-\sqrt{1-\alpha^{2}}\left|\eta_{1}^{F}\right\rangle, \\
\alpha & =\cos \left(-10.1^{\circ}\right) .
\end{aligned}
$$

On the contrary, in the vector meson sector, no octet state is observed at all. There, the masses of the neutral mesons can be explained only in terms of a pure separation between strange and non-strange quarkonia according to

$$
\begin{gathered}
|\phi\rangle=-\bar{s} s, \quad|\omega\rangle=\frac{1}{\sqrt{2}}(\bar{u} u+\bar{d} d), \\
\rho^{0}=\frac{1}{\sqrt{2}}(\bar{u} u-\bar{d} d) .
\end{gathered}
$$

It is one of the purposes of this study to understand this contradiction. We first show below that the flavor wave functions of the neutral vector 
mesons are naturally obtained as $\mathrm{U}(4)_{F}$ Weyl states in the limit of frozen charm quark degrees of freedom rather than as $\mathrm{SU}(3)_{F}$ states.

Indeed, in such a case, one has to consider the three-flavor space to emerge from truncating the four-flavor one, $q_{4}$, by treating the $c$ quark as a spectator. The new Weyl generators in the four-flavor space following from Eq. (7) read [11]:

$$
\begin{array}{ll}
E_{11}=\operatorname{diag}(1,-1,0,0), & E_{22}=\operatorname{diag}(0,1,-1,0), \\
E_{33}=\operatorname{diag}(0,0,1,-1), & q_{4}=\operatorname{column}(u, d, c, s) .
\end{array}
$$

In doing so, one finds following representations of the (orthonormalized) neutral vector meson states in the Weyl basis:

$$
\begin{aligned}
&|\phi\rangle=\lim _{m_{c} \rightarrow \Lambda_{c}} \bar{q}_{4} E_{33} q_{4},\left|\rho_{0}\right\rangle=\sqrt{2} \bar{q}_{4} \frac{E_{11}}{2} q_{4}, \\
&|\omega\rangle=\sqrt{2} \bar{q}_{4} \frac{\mathbb{1}_{2}}{2} q_{4}, \quad \mathbb{1}_{2}=\operatorname{diag}(1,1,0,0) .
\end{aligned}
$$

Here the cut off parameter $\Lambda_{c}$ has to be sufficiently large in order to ensure negligible $c$ quark effects on the $1 \mathrm{GeV}$ scale, but still finite in order to preserve the anomaly free character of the four-flavor theory [4, 12]. Note, that $E_{11}$ is nothing but twice the third component of isospin, $t_{3}^{I}$, within the first quark generation,

$$
t_{3}^{I}=\frac{E_{11}}{2}
$$

We further wish to introduce the quantity

$$
t_{3}^{H}=\frac{E_{33}}{2},
$$

to be called hyperspin in the following, which is represented by the same matrix as weak isospin for the second quark generation. As compared to the Weyl basis, the structure of the neutral vector mesons within Gell-Mann's flavor basis appears more complicated as one is forced to artificially introduce the so called 'magic', or, ideal, mixing angle $\theta$ according to 10]

$$
\begin{aligned}
|\phi\rangle & =\cos \theta \sqrt{2} \bar{q}_{3} \frac{\lambda^{8}}{2} q_{3}-\sin \theta \frac{1}{\sqrt{3}} \bar{q}_{3} \mathbb{1}_{3} q_{3}, \\
\theta & =35.3^{\circ} .
\end{aligned}
$$

The last equation clearly illustrates that the 'magic' of the ideal mixing angle is to restore Weyl's four-flavor basis by properly back rotating the Gell-Mann's one. 
On the first glance, the structure of the pseudoscalar and axial vector mesons seems to speak in favor of the octet way $\operatorname{su}(3)_{F}$ algebra. On the contrary, the vector mesons evidently prefer the heavy $c$ quark limit of $\mathrm{U}(4)_{F}$ in the limit of frozen charm degree of freedom. Simultaneously, one immediately realizes that while in the latter case the OZI is respected, it is strongly violated in the former. Indeed, the physical $\eta$ meson state in Eq. (10) can alternatively be reexpressed as a mixture of strange $(\bar{s} s)$ and non-strange $(\bar{u} u+\bar{d} d)$ quarkonia according to

$$
|\eta\rangle=\cos \epsilon(-\bar{s} s)+\sin \epsilon \frac{1}{\sqrt{2}}(\bar{u} u+\bar{d} d), \quad \epsilon=45.3^{\circ} .
$$

The last equation apparently violates the OZI rule [7] which prescribes the separation between strange and non-strange quarkonia. The reason is, that disconnected planar quark diagrams containing, say, strange to non-strange $\bar{s} s \rightarrow \bar{u} u$ quarkonia transitions are suppressed relative $\bar{s} s \rightarrow \bar{s} u+\bar{u} s$ quark recombination diagrams. This follows from gluon gauge dynamics, where the transitions between quarkonia from the first to the second or third quark generations are found to proceed over multi-gluon exchanges, while the recombination of a quark into two quark-antiquark pairs proceeds over single gluon exchange 13]. Also for the axial vector mesons $\mathrm{f}_{1}$ the OZI rule appears significantly violated. There, an angle of $\epsilon \approx-17^{\circ}$ has been reported [14. In contrast to the pseudoscalar and axial vector mesons, Eq. (13) shows that the OZI rule is respected by the vector mesons. The reason for that lies in the anomaly free character of the vector meson sector. In contrast to that, in the pseudoscalar meson sector, the anomalous divergency of the axial $\mathrm{U}(1)_{A}$ baryon number current [15] destroys the validity of the OZI rule, a topic analyzed among others in [16]. The considerations given above lead necessarily to the insight that Gell-Mann's three-flavor symmetry of hadrons is inconsistent with the OZI rule and thereby with the underlying color gauge dynamics. One is forced to note that, paradoxically, Gell-Mann's choice for the $\mathrm{su}(3)_{F}$ algebra appears more suited for describing the structure of the mesons in the anomalous axial current sector rather than in the anomaly free vector current one. In connection with that, the natural question regarding the hierarchy of the symmetries arises.

We here take the position that color gauge symmetry has to be treated as superior over constituent flavor symmetry and require the three-flavor symmetry of hadrons to respect the OZI rule. Mixing up strange with nonstrange quarkonia has to emerge within this scheme through effects violating the OZI rule, such like the effect of the 't Hooft anomaly on the structure 
of the pseudoscalar and axial vector mesons.

To get an insight into the symmetry of the required properties we consider the flavor structure of the anomaly free electromagnetic quark current $j_{\mu}$ defined as

$$
\begin{aligned}
j_{\mu} & =\frac{2}{3} \bar{u} \gamma_{\mu} u-\frac{1}{3} \bar{d} \gamma_{\mu} d+\frac{2}{3} \bar{c} \gamma_{\mu} c-\frac{1}{3} \bar{s} \gamma_{\mu} s \\
& =\bar{q}_{4} \hat{t}_{3}^{I} \gamma_{\mu} q_{4}+\frac{1}{2} \bar{q}_{4} \hat{Y} \gamma_{\mu} q_{4}, \quad q_{4}=(u d c s)^{T} .
\end{aligned}
$$

Here, we defined $\hat{Y}$ as the genuine hypercharge operator

$$
\begin{aligned}
\hat{Y} & =E_{33}+\hat{B} \\
E_{33} & =\left(\begin{array}{cccc}
0 & 0 & 0 & 0 \\
0 & 0 & 0 & 0 \\
0 & 0 & \hat{C} & 0 \\
0 & 0 & 0 & \hat{S}
\end{array}\right), \quad \hat{B}=\frac{\mathbb{1}_{(4)}}{3},
\end{aligned}
$$

where the standard notation $\hat{B}$ has been introduced for the baryon number operator. It is remarkable that the Weyl's element $\mathrm{E}_{33}$ can be identified with the difference between the hypercharge and baryon numbers as its action on the fundamental quark quadruplet $q_{4}$ reproduces charm $(C)$ and strangeness $(S)$ quantum numbers of the quarks, once use has been made of the relations $\hat{C}\left(q_{4}\right)_{i}=\delta_{i 3} c$ and $\hat{S}\left(q_{4}\right)_{j}=\delta_{j 4} s$. From Eq. (18) one reads off that the four-flavor electromagnetic quark current emerges as a Noether current with respect to the transformation

$$
q_{4} \rightarrow \exp \left(\alpha\left(t_{3}^{I}+t_{3}^{H}+B / 2\right)\right) q_{4},
$$

corresponding to an element of the $\mathrm{su}(2)_{u d} \otimes \mathrm{su}(2)_{c s} \otimes \mathrm{u}(1)$ subalgebra of the full group $\mathrm{U}(4)_{F}$ considered in the heavy $c$ quark limit. This observation underlines once more the importance of the $\mathrm{U}(4)_{F}$ group for hadron dynamics. Remarkably, despite the fact, that four-flavor symmetry is more badly broken than the three flavor one, as the $c$ quark is much heavier than the others, the structure of hadrons and the transformation properties of the currents are nonetheless determined by the representations of that very symmetry [10]. Consequently, three-flavor currents have to be considered to emerge from a flavor space containing complete quark generations after freezing out the heavy quarks degrees of freedom rather than as primordial $\mathrm{SU}(3)_{F}$ quantities thus requiring a revision of the traditional viewpoint on 
$\mathrm{SU}(4)_{F}$ as a mathematical exercise [17]. Now, as long as $j_{\mu}$ is conserved, its total charge

$$
Q(t)=\int j_{0}(t, \vec{x}) \mathrm{d}^{3} \vec{x},
$$

is a constant of motion and labels the hadron states. When considered as an operator, $\hat{Q}$ is directly read off from Eq. (18) to be related to the operators of isospin $\hat{t}_{3}^{I}$ and hypercharge $\hat{Y}$ via the four-flavor version of the Gell-Mann-Nakano-Nishijima relation [18]

$$
\begin{aligned}
\hat{Q} & =\hat{t}_{3}^{I}+\frac{1}{2} \hat{Y} \\
& =\hat{t}_{3}^{I}+\hat{t}_{3}^{H}+\frac{1}{2} \hat{B} .
\end{aligned}
$$

Now, in the heavy $c$ quark limit of Eq. (22), where the charm degree of freedom is frozen out, one finds the expression for the hypercharge in the truncated three-flavor space as

$$
\begin{aligned}
\lim _{m_{c} \rightarrow \Lambda_{c}}\left(\hat{C}+\hat{S}+\frac{1}{3} \mathbb{1}_{(4)}\right)=\hat{S}_{(3)}+\frac{1}{3} \mathbb{1}_{(3)} & \equiv \frac{1}{\sqrt{3} \lambda^{8}} \\
\hat{S}_{3} & =\left(\begin{array}{ccc}
0 & 0 & 0 \\
0 & 0 & 0 \\
0 & 0 & -1
\end{array}\right) .
\end{aligned}
$$

The matrix $\hat{S}_{(3)}+\frac{1}{3} \mathbb{1}_{3}$, on the r.h.s. of Eq. (23), representing the hypercharge in the truncated flavor space (now three dimensional) happens by accident to be traceless despite the fact that the full four-flavor hypercharge matrix $\hat{Y}$ in Eq. (19) is not and coincides by chance numerically with $\frac{1}{\sqrt{3}} \lambda^{8}$. In this way the confusing impression appears that hypercharge can be introduced on the level of the special group $\mathrm{SU}(3)_{F}$ while it is a property of the full $\mathrm{U}(4)_{F}=\mathrm{SU}(4)_{F} \otimes \mathrm{U}(1)$ group (see [9]) for more details).

The physically realized decomposition of $Q$ in Eq. (22) suggests the precise three-flavor symmetry of hadrons to be the heavy charm quark limit of the subgroup $\mathrm{SU}(2)_{u d} \otimes \mathrm{SU}(2)_{c s} \otimes \mathrm{U}(1)$ of $\mathrm{U}(4)_{F}$ rather than Gell-Mann's $\mathrm{SU}(3)_{F}$. In the following, we will denote this group by $S_{O Z I}$ and consider it to be the precise three-flavor symmetry of hadrons, i.e.

$$
S_{O Z I}=\lim _{m_{c} \rightarrow \Lambda_{c}} S U(2)_{u d} \otimes S U(2)_{c s} \otimes U(1) .
$$

A value of $\Lambda_{c} \approx 1.5 \mathrm{GeV}$ seems reasonable in that respect. The big advantage of $\mathrm{S}_{O Z I}$ is that it allowed us to define the quantum number of 
the hypercharge in Eq. (22) in a way consistent with the four-flavor GellMann-Nakano-Nishijima relation, which, in being a $\mathrm{SU}(4) \otimes \mathrm{U}(1)$ quantity is undefinable within the concept of a special unitary group. Now, in contrast to $Q$, the axial flavor rotations corresponding to Eq. (23)

$$
q_{3}^{\prime}=e^{i \beta\left(\hat{S}_{3}+\frac{1}{3} \mathbb{1}_{3}\right) \gamma_{5}} q_{3},
$$

cannot create a conserved charge as the term containing the unit element in the exponent in Eq. (25) will give rise to the anomalously divergent axial $\mathrm{U}(1)_{A}$ baryon number current, for which no chiral limit can be formulated. As a consequence, the full hypercharge axial current will be anomalous too. Once more, the anomaly does not disappear in going from the four-flavor to the truncated three-flavor space. The only way to obtain in the chiral limit of vanishing quark masses a conserved, and therefore observable neutral flavor axial current, is to suppress the $\mathrm{U}(1)_{A}$ transformation. In doing so, one finds the following neutral axial current $j_{\mu, 5}$ :

$$
\begin{aligned}
j_{\mu, 5} & =\bar{q}_{4} \gamma_{\mu} \gamma_{5}\left(\hat{t}_{3}^{I}+\frac{1}{2}(\hat{Y}-\hat{B})\right) q_{4} \\
& =\frac{1}{2} \bar{u} \gamma_{\mu} \gamma_{5} u-\frac{1}{2} \bar{d} \gamma_{\mu} \gamma_{5} d+\frac{1}{2} \bar{c} \gamma_{\mu} \gamma_{5} c-\frac{1}{2} \bar{s} \gamma_{\mu} \gamma_{5} s .
\end{aligned}
$$

The flavor structure of $j_{\mu, 5}$ in the last equation, which can be partially conserved now, reflects the exclusion of the anomalous $\mathrm{U}(1)_{A}$ current which can not be used any longer as a building block for the construction of an anomaly free octet axial current. Remarkably, $j_{\mu, 5}$ respects the OkuboZweig-Iizuka (OZI) rule [7], and its structure is identical (up to the factor of $-1 / 2$ ) to that of the neutral weak axial vector current. For this reason, the well established universality of the flavor changing weak and strong axial vector currents underlying the current algebra can be extended to include the neutral ones.

The $j_{\mu, 5}$ current decomposes in the four-flavor space into an isovector $\left(j_{\mu, 5}^{I}\right)$ and a purely strange $\mathrm{SU}(2)_{I}$ isosinglet $\left(j_{\mu, 5}^{c s}\right)$ component while a hypercharge component is absent [11],

$$
\begin{gathered}
j_{\mu, 5} \rightarrow j_{\mu, 5}^{I}+j_{\mu, 5}^{c s}, \\
j_{\mu, 5}^{I}=\bar{q}_{4} \frac{E_{11}}{2} \gamma_{\mu} \gamma_{5} q_{4}, \quad j_{\mu, 5}^{c s}=\frac{1}{2} \bar{q}_{4} \gamma_{\mu} \gamma_{5} \frac{E_{33}}{2} q_{4} .
\end{gathered}
$$

A different decomposition of $j_{\mu, 5}$ can be performed in the $\mathrm{V}$-spin basis of the $(u, s)$ quark doublet. It is easily obtained from the last equation through 
the replacements $E_{11} \rightarrow E_{11}+E_{22}+E_{33}$, and $s \rightarrow d$, as

$$
\begin{gathered}
j_{\mu, 5} \rightarrow j_{\mu, 5}^{V}+j_{\mu, 5}^{c d}, \\
j_{\mu, 5}^{V}=\bar{q}_{4} \frac{E_{11}+E_{22}+E_{33}}{2} \gamma_{\mu} \gamma_{5} q_{4}, \quad j_{\mu, 5}^{c d}=\bar{q}_{4} \gamma_{\mu} \gamma_{5} \frac{-E_{22}}{2} q_{4} .
\end{gathered}
$$

Finally, in the U-spin basis of the $(d, s)$ quark doublet, $j_{\mu, 5}$ transforms according a representation of $\mathrm{u}(2)_{u d} \otimes \mathrm{su}(2)_{d s}$ rather than, as in the cases before, according to the direct product of two special unitary representations:

$$
\begin{aligned}
j_{\mu, 5} \rightarrow & 2 j_{\mu, 5}^{I}+j_{\mu, 5}^{U}-j_{\mu, 5}^{c u} \\
j_{\mu, 5}^{U}=\bar{q} \frac{E_{22}+E_{33}}{2} \gamma_{\mu} \gamma_{5} q, & j_{\mu, 5}^{c u}=\bar{q} \frac{E_{11}+E_{22}}{2} \gamma_{\mu} \gamma_{5} q .
\end{aligned}
$$

Now, in the limit of frozen charm degree of freedom, the purely charmed currents from Eqs. (27)-(29) drop out, and one finds three different possibilities to embed a two-flavor group within the restricted $\mathrm{U}(4)_{F}$ group. These are the groups $\mathrm{SU}(2)_{V}, \mathrm{SU}(2)_{U}$, and $\mathrm{U}(2)_{I}$ with $\mathrm{U}(2)_{I}=\mathrm{SU}(2)_{I} \otimes \mathrm{U}(1)$, in turn corresponding to $\mathrm{V}$-spin, U-spin, and isospin. Therefore, in the case of a spectator $c$ quark, four-flavor chiral symmetry $\mathrm{U}(4)^{L} \otimes \mathrm{U}(4)^{R}$ restricted to $\left[\mathrm{SU}(2)_{u d}^{L} \otimes \mathrm{SU}(2)_{c s}^{L} \otimes \mathrm{U}^{L}(1)\right] \otimes\left[\mathrm{SU}(2)_{u d}^{R} \otimes \mathrm{SU}(2)_{c s}^{R} \otimes \mathrm{U}^{R}(1)\right]$ can be spontaneously reduced to either $\mathrm{U}(2)_{I}, \mathrm{SU}(2)_{V}$, or $\mathrm{SU}(2)_{U}$ symmetries. In the first case, the associated Goldstone bosons are the three pions and the entirely non-strange isosinglet meson, $\eta_{1}^{I}$ from Eq. (8), while in the second and third cases one finds such to be the two charged $\left(K^{+}\right.$and $\left.K^{-}\right)$, and two neutral $\left(K^{0}\right.$, and $\left.\bar{K}^{0}\right)$ kaons, respectively. The broken generators $t_{3}^{U}$ and $t_{3}^{V}$ will share as a common neutral Goldstone boson the strange meson $\left|\eta^{s}\right\rangle=-\bar{s} s$, the only neutral state that appears orthogonal to both $\pi^{0}$ and $\eta_{1}^{I}$, respectively. In case the OZI rule was respected by the pseudoscalar mesons, the required strange Goldstone boson would be $\eta^{s}$, the analogue to the strange $\phi$ meson from the vector meson nonet.

However, due to the influence of the $\mathrm{U}(1)_{A}$ anomaly on the spectroscopic level, the OZI rule is violated for the particular case of the pseudoscalar and axial vector mesons and the strange and non-strange quarkonia are mixed up [16]. This is manifest in Eq. (10) by means the deviation of the wave function of the physical $\eta$ meson state from the $(-\bar{s} s)$ one. Eq. (10) clearly illustrates that the physical $\eta$ meson transforms according to a representation of $\lim _{m_{c} \rightarrow \Lambda_{c}} \mathrm{SU}(2)_{c s} \otimes \mathrm{U}(1)_{I}$ rather than as a genuine $\mathrm{SU}(3)_{F}$ state. From this point of view, the significant non-strange quarkonium component of the $\eta$ meson wave function appears as an artifact of the violation of the 
OZI rule for the pseudoscalar mesons as attributed to the $\mathrm{U}(1)_{A}$ anomaly rather than as a consequence of an underlying fundamental $\mathrm{SU}(3)_{F}$ symmetry. Nonetheless, through the significant $\bar{s} s$ component in its wave function, the $\eta$ meson is still able to meet its purpose as the 'strange' Goldstone boson mentioned above.

\section{Summary}

To summarize, we pointed out that the structure of the vector mesons and their currents, in respecting the fundamental gluon gauge dynamics by means of the OZI rule, requires four-flavor symmetry of hadrons to be realized in the Weyl basis and contradicts thereby Gell-Mann's eightfold way concept. We deduced the flavor symmetry of hadrons on the grounds of the transformation properties of the fundamental electromagnetic current in a flavor space containing two complete quark generations with the heaviest charm flavor being frozen out. We concluded that the only three-flavor symmetry of hadrons of the required properties is $\mathrm{S}_{O Z I}$ in Eq. (24) rather than Gell-Mann's $\mathrm{SU}(3)_{F}$. We pointed out that the difference between the two schemes is revealed by the properties of the non-isovector part of the neutral axial current. The latter is identical to the hypercharge axial current only for the case of the $S_{O Z I}$ symmetry and contains the 't Hooft anomaly. Within the $\mathrm{S}_{O Z I}$ symmetry scenario, the only isoscalar axial current having a well defined chiral limit is $\lim _{m_{c} \rightarrow \Lambda_{c}} J_{\mu, 5}^{c s}$ and purely flavored. On the contrary, the non-isotriplet part of the neutral axial current within the $\mathrm{SU}(3)_{F}$ scheme is a F-spin scalar an if it were physically realized, it could be considered it as a partially conserved. The decision, which one of the two threeflavor symmetries is realized in nature, can be made only through a suited comparison with data. The physical observable which is sensitive to the nonisotriplet neutral axial current, is the $\eta N$ coupling constant. In the case of a (spontaneously broken) $\mathrm{SU}(3)_{\otimes} \mathrm{SU}(3)_{R}$ chiral symmetry, the $\eta N$ coupling is determined through the octet Goldberger-Treiman relation and appears proportional to the octet axial coupling constant $g_{A}^{8}=\frac{1}{\sqrt{3}}(\Delta u+\Delta d-2 \Delta s)$ with $\Delta q_{i}$ standing for the polarization of the $q_{i}$ quark sea. The value of $g_{A}^{8}$ can slightly be reduced by accounting for the $\eta-\eta^{\prime}$ mixing but this reduction is still not sufficient to explain data. Within the $\mathrm{S}_{O Z I}^{L} \otimes \mathrm{S}_{O Z I}^{R}$ symmetry scenario, where a purely flavored isosinglet current comes in place of the octet axial current, the (tree-level) couplings of the $\eta$ and $f_{1}(1285)$ mesons to the nucleon will be proportional to $\lim _{\Delta c \rightarrow 0}(\Delta c-\Delta s)$, the negligible fraction of 
the nucleon helicity carried by the flavored quark sea. This may be the main reason for the observed strong suppression of the $\eta N$ coupling relative the value predicted by the octet Goldberger-Treiman relation [6]. To explain the small, but non-negligible $\eta N N$ and $f_{1} N N$ vertices, triangular vertices of the type $a_{0} \pi N$ and $K K^{*} Y$, respectively, have to be considered. Vertex loop corrections of that type have been calculated, for example, in [19], and shown to be important for data interpretation.

In case of the $S_{O Z I}$ symmetry and in the absence of an anomaly, the $\eta$ boson would appear as a purely strange quarkonium and would act as the analogue of the $\phi$ vector meson. That this is not the case is entirely due to the 't Hooft anomaly. Within the context of the $S_{O Z I}$ symmetry, the strong presence of non-strange quarkonium components in the wave function of the $\eta$ meson appeared as an artifact of the violation of the OZI rule through the $\mathrm{U}(1)_{A}$ anomaly rather than as the consequence of an underlying fundamental $\mathrm{SU}(3)_{F}$ symmetry. The consequence of the new symmetry scheme presented above is that the spontaneous breaking of chiral symmetry of the QCD lagrangian will now proceed over the chain

$$
\begin{aligned}
\lim _{m_{c} \rightarrow \Lambda_{c}}\left([ S U ( 2 ) _ { u d } ^ { L } \otimes S U ( 2 ) _ { c s } ^ { L } ] \otimes \left[S U(2)_{u d}^{R} \otimes\right.\right. & \left.S U(2)_{c s}^{R}\right] / \\
& S U(2)_{I, V, U},
\end{aligned}
$$

rather than over $S U(3)^{L} \otimes S U(3)^{R} / S U(3)_{F}$. The charged Goldstone bosons associated with Eq. (30) will be the three pions and the four kaons, while an entirely strange, neutral eight Goldstone boson, is absent but its role can still be played by the strange quarkonium component of the $\eta$ meson.

Work supported partly by CONACyT Mexico, and partly by the Deutsche Forschungsgemeinschaft (SFB 201).

\section{References}

[1] G. Zweig, CERN Report No. 8182/TH 401 (1964).

[2] M. Gell-Mann, Phys. Lett. 8 (1964) 214.

[3] M. Kirchbach, Mod. Phys. Lett. A12 (1997) 2373, 3177; ibid. A13 (1998) 813.

[4] S. Weinberg, The Quantum Theory of Fields, (Cambridge Univ. Press, 1996) pp.225-234. 
[5] S. A. Coon and M. D. Scadron, Phys. Rev. C42 (1990) 2256.

[6] M. Kirchbach and H. -J. Weber, Comm. Nucl. Part. Phys. 22 (1998) 171.

[7] S. Okubo, Phys. Lett. 5 (1963) 163.

G. Zweig, CERN Report 8419/TH 412 (1964).

J. Iizuka, Progr. Theor. Phys. Suppl. No. 37-38 (1966) 21.

[8] M. Kaku, Quantum Field Theory, (Oxford Univ. Press, New York, 1993) pp. 371-374.

[9] J. F. Cornwell, Group Theory in Physics , (Techniques in Physics, 1984).

[10] Review of Particle Physics, Eur. Phys. J. C3 (1998) pp. 109-110.

[11] M. Kirchbach. Phys. Rev. D58 (1998) 117901.

[12] C. D. Frogatt and H. B. Nielsen, in The Origin of Symmetry (World Scientific, Singapore) 1991, pp. 32-34.

[13] R. K. Bhaduri, Models of the Nucleon (Addison-Wesley, 1988).

[14] T. Bolton et al. Phys. Lett. B228 (1992) 495.

[15] G. 't Hooft, Phys. Rev. D14 (1976) 3432.

[16] S. Forte and E. Shuryak, Nucl. Phys. B 367 (1991) 153.

[17] H. Georgi, Lie Algebras in Particle Physics, (The Benjamin/Cummings Publ. Comp. Inc. , Reading, Massachusetts) 1982, pp. 154-155.

[18] M. Gell-Mann, Phys. Rev. 92 (1953) 833.

T. Nakano and K. Nishijima, Progr. Theoret. Phys. 10 (1953) 581.

[19] M. Kirchbach and L. Tiator, Nucl. Phys. A604 (1996) 385.

S. Neumeier and M. Kirchbach, MKPH-T-98-13 